\title{
AC Susceptibility of YBCO 1:2:3 Films on Silver Substrates
}

\author{
R. ZAlecki, W.M. WOCH*, M. Chrobak and A. KoŁODZIEJCZYK \\ Solid State Physics Department, Faculty of Physics and Applied Computer Science \\ AGH University of Science and Technology, Krakow, Poland
}

\begin{abstract}
The $\mathrm{YBa}_{2} \mathrm{Cu}_{3} \mathrm{O}_{\delta}$ films were obtained directly on silver substrates by the sedimentation processes. The thicknesses of these films are of the order of several tens $\mu$. The temperature dependences of the AC susceptibility of the films were measured and analyzed. The critical temperatures of these specimens obtained from the dispersion part of AC susceptibility vary from $89.2 \mathrm{~K}$ to $92.5 \mathrm{~K}$. The critical currents were calculated from the absorption part of AC susceptibility using the Bean model. The temperature dependences of the critical currents were fitted using the Ginzburg-Landau strong coupling limit approach.
\end{abstract}

DOI: 10.12693/APhysPolA.126.A-13

PACS: 74.72.-h, 74.25.F-, 74.25.Ha, 74.25.Sv

\section{Introduction}

The preparation of high temperature superconductors (HTS) films has gained much interest for large-scale applications including power system components such as electrical motors, generators, power transmission cable, transformers, and superconducting magnetic energy storage devices. Extensive investigations have been connected to optimizing the fabrication and processing techniques of HTS wires and tapes.

Silver is typically used as a substrate of superconducting material in superconducting tapes and wires of yttrium $[1,2]$, thallium $[3,4]$ or bismuth $[5,6]$ based high temperature superconductors. The direct deposition of YBCO films on silver substrates without any buffer layers is one of the most promising and low-cost method of production of the superconducting tapes [7]. The silver turns out to be only a metal on which YBCO material can be deposited without buffer layers because it does not react with the YBCO and fills the pores and empty spaces between the grains [8-10]. However, the silver substrate may influence on the deposited superconducting material due to sublimation processes of a silver at high sintering temperatures as well as due to crystal mosaics which are formed on a silver surface at high temperatures. This problem is particularly important in the case of YBCO high temperature superconductors because they require high sintering temperatures up to $950^{\circ} \mathrm{C}$ to obtain good superconducting quality properties.

We present results of AC susceptibility measurements of YBCO thick films deposited on silver substrates using the sedimentation process and then sintered at temperature of $900{ }^{\circ} \mathrm{C}$. The thickness of the films were 120,113 , 85 , and $20 \mu \mathrm{m}$, respectively. The temperature dependences of the absorption part of AC susceptibility were carried out to evaluate the critical current densities using the Bean critical state model.

\section{Experimental}

Polycrystalline YBCO samples were prepared with the standard powder solid-state reaction technique. It consists of mixing of the copper and yttrium oxides and the barium carbonate in appropriate amounts. The mixed powders were sintered at $940^{\circ} \mathrm{C}$ and then reground into fine powder and pressed to the pellet. The pellet was sintered at $940^{\circ} \mathrm{C}$ in flowing oxygen atmosphere and then cooled down slowly to the room temperature. The pellet was reground. The obtained powder was mixed in the isopropanol to make the suspension. The starting suspension after ten seconds of sedimentation process was poured to another vessel. The powder left on the bottom of the first vessel contains the largest grains (it was called as the first fraction). This procedure was repeated for the rest suspension to obtain the smaller grains fractions. The second fraction was obtained after one minute of sedimentation, the third fraction - after one hour of sedimentation and the fourth - after eighteen hours of sedimentation. The powders of each fraction were mixed separately with isopropanol in a vessel with a silver substrate on its bottom. After evaporation of the alcohol the powder deposited on the silver substrate was pressed with the pressure of about $1 \mathrm{GPa}$. The films were put together into furnace and sintered at $900{ }^{\circ} \mathrm{C}$ for $2 \mathrm{~h}$ and then cooled down to $400{ }^{\circ} \mathrm{C}$ in the flowing oxygen atmosphere during $5 \mathrm{~h}$. Finally, they were left at this temperature for $2 \mathrm{~h}$ for oxidation and then they were cooled down slowly to the room temperature. The thickness of the films with the powders of the first to the fourth fraction were measured using XRF spectrometer and they were 120, 113, 85 , and $20 \mu \mathrm{m}$, respectively.

The real and imaginary parts of AC susceptibility as a function of temperature and magnetic field amplitude were measured by a standard mutual inductance bridge operating at the frequency of $189 \mathrm{~Hz}$. A Stanford SR 830 lock-in nanovoltmeter served both as a source for the AC current for the coil which produced the AC magnetic field and as a voltmeter of the bridge.

\footnotetext{
${ }^{*}$ corresponding author; e-mail: wmwoch@agh.edu.pl
} 


\section{Results}

The AC susceptibility measurements of YBCO films deposited on the silver substrates are shown in Figs. 1-4. The figures concern the films obtained from the YBCO powder of the fractions $1-4$, respectively. The measurements carried out at different AC field amplitudes show a good quality of the superconducting material. The intragrain critical temperatures obtained from the temperature dependences of dispersion part of the AC susceptibility [11] are as follows: $92.5 \mathrm{~K}, 92.5 \mathrm{~K}, 90.8 \mathrm{~K}$ and $89.2 \mathrm{~K}$, respectively for the fractions $1-4$. The dispersion $\left(\chi^{\prime}\right)$ and the absorption $\left(\chi^{\prime \prime}\right)$ parts of the AC susceptibility measurements convince that the films do not form separate superconducting islands but they are superconducting in the whole volume. The absorption part of the $\mathrm{AC}$ susceptibility exhibits in general one peak, concerning to the energy losses connected with the magnetic field penetration into the inter-granular regions. The $\chi^{\prime \prime}$ peaks move fast to the lower temperatures when the applied magnetic field is being increased. No absorption peak often observed in the ceramic samples close to the critical temperature (connected with the absorption processes in the grains) was observed. The temperature dependences of the dispersion parts of the AC susceptibility do not depend on the AC magnetic field amplitude in the vicinity of the critical temperature and these curves are being split at the temperature below about $90.3 \mathrm{~K}$.
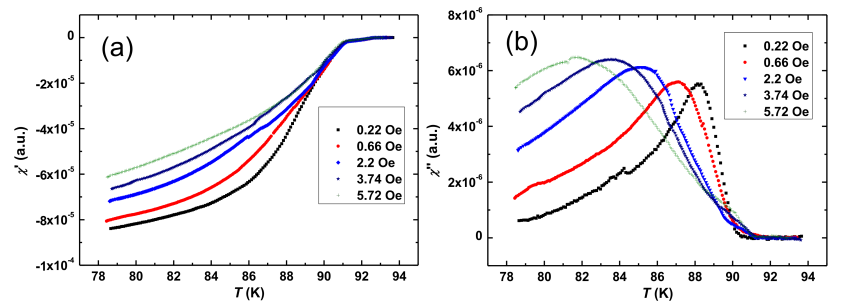

Fig. 1. Dispersive (a) and absorption (b) components of the susceptibility of the YBCO film made from the first powder fraction deposited on the silver substrate.
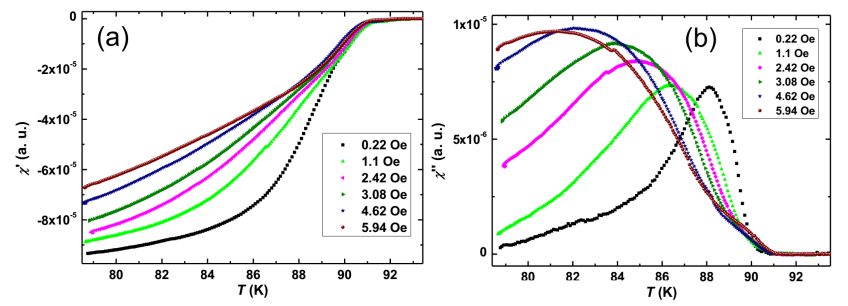

Fig. 2. As in Fig. 1, but for the second powder fraction.

These measurements show that we do not lose superconducting properties and the critical temperature in consequence of deposition superconducting powder onto Ag surface and sintering them together. From the susceptibility measurements the intergrain critical temperature defined at the maximum $\chi^{\prime \prime}\left(H_{\mathrm{AC}} \rightarrow 0\right)$ is about
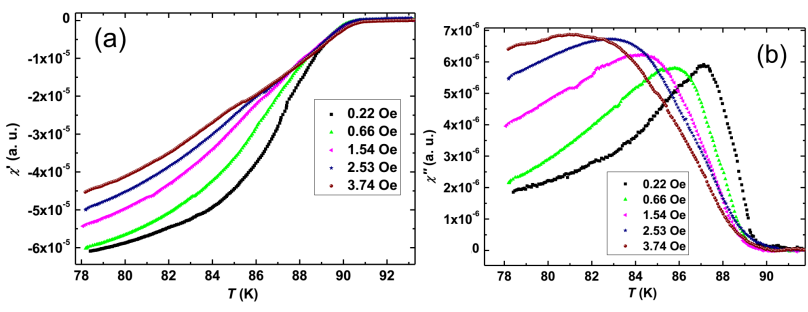

Fig. 3. As in Fig. 1, but for the third powder fraction.
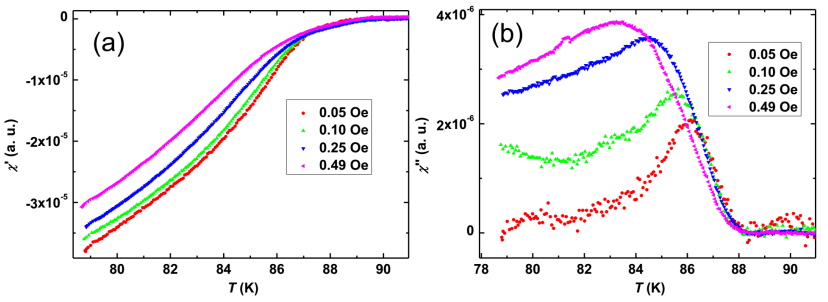

Fig. 4. As in Fig. 1, but for the fourth powder fraction.

88-89 $\mathrm{K}$ while the intra-grain critical temperature defined for $\chi^{\prime}=0$ is only about $1-3 \mathrm{~K}$ higher.

The temperature dependences of the absorption part of $\mathrm{AC}$ susceptibility at different magnetic field $H_{\mathrm{AC}}$ amplitudes were measured to evaluate the critical current densities of the films. They were obtained from the peak position of the absorption part of susceptibility within the Bean critical state model from the formula $[12,13]$ :

$$
J_{c}=\frac{2 H_{\mathrm{AC}}}{d},
$$

where $H_{\mathrm{AC}}$ is the $\mathrm{AC}$ magnetic field amplitude and $d$ is the sample thickness in the perpendicular direction to the $\mathrm{AC}$ field. The results of inter-grain critical current densities calculations for the films of each fraction of the YBCO powder are presented in Fig. 5.

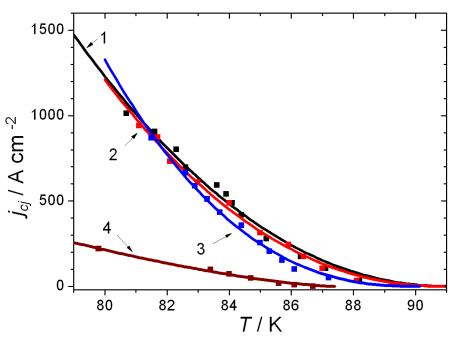

Fig. 5. Critical current densities of the YBCO films made from the fractions 1-4 powders deposited on the silver substrates. The data points were fitted with the formula (2) in which the $J_{\mathrm{c} 0}, T_{\mathrm{c}}$ and $n$ are the fit parameters.

The critical current flows through the inter-grain links which may be treated as the Josephson junctions (weak links). The critical current is limited by the weakest junctions on the percolate path of the current. According to the Ginzburg-Landau strong coupling limit approach, the critical current varies with temperature as shown in the following equation [14]: 


$$
J_{\mathrm{c}}=J_{\mathrm{c} 0}\left(1-\frac{T}{T_{\mathrm{c}}}\right)^{n},
$$

where $T_{\mathrm{c}}$ is the critical temperature that depends on the applied magnetic field and $J_{\mathrm{c} 0}$ is the critical current at $0 \mathrm{~K}$. Originally, the exponent $n$ was determined to be $1.5[15]$ but it may vary within a wide range [16]. If the exponent $n$ is greater than unity there is strong pinning and a vortex glass structure exists. In this case the function expressed by Eq. (2) has an upward curvature which is typical for HTS. If the exponent $n$ is less than unity, the pinning force is weak and limits the critical current. Then the function expressed by Eq. (2) has a downward curvature typical for low temperature superconductors.

TABLE

Thickness $\delta$, critical current densities at $77 \mathrm{~K}$, critical temperatures $T_{\mathrm{c}}$ and exponents $n$ of the YBCO films obtained from the relevant grain fractions.

\begin{tabular}{l|c|c|c|c}
\hline \hline Sample & $\delta[\mu \mathrm{m}]$ & $J_{\mathrm{c}}(77 \mathrm{~K})\left[\frac{\mathrm{A}}{\mathrm{cm}^{2}}\right]$ & $T_{\mathrm{c}}[\mathrm{K}]$ & $n$ \\
\hline fraction 1 & 120 & $1800 \pm 400$ & $88.5 \pm 0.5$ & $1.37 \pm 0.15$ \\
fraction 2 & 113 & $1800 \pm 400$ & $89.0 \pm 0.5$ & $1.54 \pm 0.20$ \\
fraction 3 & 85 & $2100 \pm 700$ & $88.2 \pm 0.4$ & $1.67 \pm 0.16$ \\
fraction 4 & 20 & $330 \pm 40$ & $86.4 \pm 0.2$ & $1.07 \pm 0.05$
\end{tabular}

The temperature dependences of the critical current densities of the films were fitted using Eq. (2) in which the critical temperature $-T_{\mathrm{c}}$, the critical current at $0 \mathrm{~K}$ - $J_{\mathrm{c} 0}$, and the exponent $n$ are the fit parameters. The solid lines that are shown in Fig. 5 present fit of Eq. (2) to the experimental data. The fit parameters: $T_{\mathrm{c}}$ and $n$ as well as the critical currents in the liquid nitrogen temperature, $J_{\mathrm{c}}(77 \mathrm{~K})$, calculated according to the formula (2) for the all YBCO films and the appropriate thickness of the samples are collected in Table. The critical currents in the liquid nitrogen temperature and the exponent $n$ obtained in this paper are a little better than obtained in our previous work [17]. From the results one can observe the existence of superconductivity up to $90 \mathrm{~K}$ for $\mathrm{YBCO}$ films except of the film made from the fourth fraction (the smallest) powder; but the critical current densities are not very high. The critical temperatures calculated from the fits are systematically (1-2 K) smaller than the temperatures obtained from the $\mathrm{AC}$ susceptibility measurements $\left(\chi^{\prime}=0\right)$.

\section{Summary}

The YBCO films deposited directly on silver substrates were obtained using the sedimentation process. The thickness of the films were 120, 113, 85, and $20 \mu \mathrm{m}$ and the intra-grain critical temperatures 92.5, 92.5, 90.8, $89.2 \mathrm{~K}$, respectively. The critical currents were calculated from the absorption part of AC susceptibility within the Bean critical state model. The highest value of the critical current $J_{\mathrm{c}}(77 \mathrm{~K})=2100 \mathrm{~A} \mathrm{~cm}^{-2}$ was found for the sample of $85 \mu \mathrm{m}$ thick made from fraction 3 . This film has also the highest exponent $n=1.67$ that confirms the strongest pinning force among other ones. One can observe that the silver substrate does not influence strongly on the properties of the YBCO films sintered at the temperature of $900^{\circ} \mathrm{C}$. The superconducting parameters are similar for the fractions 1-3 and they do not differ much from those obtained for the bulk ceramics. Only the film from the fraction 4 (20 $\mu$ m thick) has considerably worse superconducting parameters such as $J_{\mathrm{c}}$ and $T_{\mathrm{c}}$.

\section{Acknowledgments}

This work was supported by the Polish Ministry of Science and Higher Education and its grants for Scientific Research. One of us (M.Ch.) has been partly supported by the EU Human Capital Operation Program, Polish project No. POKL.04.0101-00-434/08-00.

\section{References}

[1] X. Wen, D. Qu, B.A. Tent, D. Shi, M. Tomsic, L. Cowey, M. White, IEEE Trans. Applied Supercond. 9, 1506 (1999).

[2] D. Liu, M. Zhou, X. Wang, H. Suo, T. Zuo, M. Schindl, R. Flukiger, Supercond. Sci. Technol. 14, 806 (2001).

[3] C. Deinhofer, G. Gritzner, Supercond. Sci. Technol. 17, 1196 (2004).

[4] W.M. Woch, W. Tokarz, R. Zalecki, A. Kołodziejczyk, C. Deinhofer, G. Gritzner, Supercond. Sci. Technol. 23, 025004 (2010).

[5] A.P. Malozemoff, IEEE Spectrum 30, 26 (1993).

[6] B. Ullmann, A. Gabler, M. Quilitz, W. Goldacker, IEEE Trans. Appl. Supercond. 7, 2042 (1997).

[7] Y. Ma, K. Watanabe, S. Awaji, M. Motokawa, Physica C 357-360, 337 (2001).

[8] W.M. Woch, J. Chmist, J. Przewoźnik, J. Niewolski, R. Zalecki, A. Kołodziejczyk, Mol. Phys. Rep. 34, 175 (2001).

[9] Y. Koike, Y. Yamada, I. Hirabayashi, H. Akata, K. Higashiyama, in: Advances in Superconductivity XI, Eds. N. Koshizuka, S. Tajima, Springer Japan, Tokyo 1999, p. 741.

[10] C. Zang, A. Kulpa, A.C.D. Chaklader, Physica C 252, 67 (1995).

[11] W.M. Woch, R. Zalecki, A. Kołodziejczyk, H. Sudra, G. Gritzner, Mater. Sci. (Poland) 26, 1091 (2008).

[12] C.P. Bean, Phys. Rev. Lett. 8, 250 (1962).

[13] J.R. Clem, Physica C 153-155, 50 (1988).

[14] J.R. Clem, B. Bumble, S.I. Raider, W.J. Gallagher, Y.C. Shih, Phys. Rev. B 35, 6637 (1987).

[15] K.A. Müller, M. Takashige, J. Bednorz, Phys. Rev. Lett. 58, 1143 (1987); Y. Yeshurun, A.P. Malozemoff, Phys. Rev. Lett. 60, 2202 (1988).

[16] W.M. Woch, R. Zalecki, A. Kołodziejczyk, H. Sudra, G. Gritzner, Supercond. Sci. Technol. 21, 085002 (2008).

[17] R. Zalecki, W.M. Woch, M. Chrobak, A. Kołodziejczyk, Acta Phys. Pol. A 121, 850 (2012). 